Journal of Mathematics and Statistics 6 (1): 17-22, 2010

ISSN 1549-3644

(C) 2010 Science Publications

\title{
Compare Between Two-Unit Cold Standby and Warm Standby Outdoor Electric Power Systems in Changing Weather
}

\author{
${ }^{1}$ G.S. Mokaddis, ${ }^{2}$ M. Salah EL-Sherbeny and ${ }^{3}$ Entesar Al-Esayeh \\ ${ }^{1}$ Department of Mathematics, Faculty of Science, Ain Shams University, Cairo, Egypt \\ ${ }^{2}$ Department of Mathematics, Faculty of Science, Helwan University, Cairo, Egypt \\ ${ }^{3}$ Department of Statistics, Faculty of Science, 7th April University, Zawia, Libya
}

\begin{abstract}
Problem statement: This study presents the reliability analysis of two mathematical models representing electric power systems operating in fluctuating outdoor weather (i.e., normal and stormy weather) and compared between two models. Approach: Model I deals the reliability analysis of a single-server two-unit cold standby, Model II deals the reliability analysis of a single-server two-unit warm standby, for two systems with two different modes (normal, total failure). System failure occurs when both the units fail totally. Results: The failure rate and failed repair rate of a unit are constants. Laplace transforms of the various state probabilities have been derived and then reliability is obtained by the inversion process. Conclusion/Recommendations: Mean Time To Failure (MTTF) are derived. The failure times of operating/spare units and repair time of failed units are exponential distributed. Certain important results are compared between two systems.
\end{abstract}

Key words: Reliability to system R(t) and Mean-Time To Failure (MTTF)

\section{INTRODUCTION}

Introduction of redundancy, are some of the wellknown methods by which the reliability of a system can be improved. Two-unit standby redundant systems have been extensively studied by several authors in the past. earlier study researchers (Balbir and Subramanyam, 1985) have studied the stochastic analysis of two-unit outdoor electric power systems in changing weather (Dhillon and Natesan, 1986) have studied the reliability analysis of man-machine system operating subject to physical conditions (Kuo-Hsiung Wang et al., 2006) have studied the Comparison of reliability and the availability between four systems with warm standby components and standby switching failure (Mokaddis et al., 2009) have studied the stochastic behavior of a two-unit warm standby system with two types of repairmen and patience time.

This study presents two mathematical models representing electric power systems operating in fluctuating outdoor weather (i.e., normal and stormy weather).

Model I represents a repairable two identical unit system with one unit in operation and the other one in cold standby mode. The system operates in the changing environments (i.e., normal and stormy weather). The failed units are repairable. The system ceases to function when both the units are non-operative. The system state transition diagram is shown in Fig. 1.

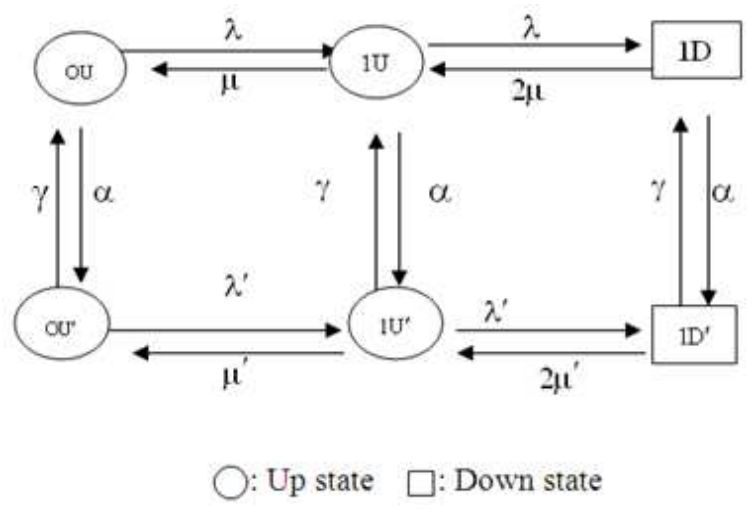

Fig. 1: State transition diagram

Model II essentially represents the same system configuration as in model I but with one exception that the unit standby is warm. The state space diagram of the system is shown in Fig. 2. Certain important results have been derived as compared between two models and. Some numerical results for the mean times to failure are calculated.

The following assumptions are adopted: The following assumptions are associated with Models I and II:

- Unit failure rate is constant

Corresponding Author: G.S. Mokaddis, Department of Mathematics, Faculty of Science, Ain shams University, Cairo, Egypt 


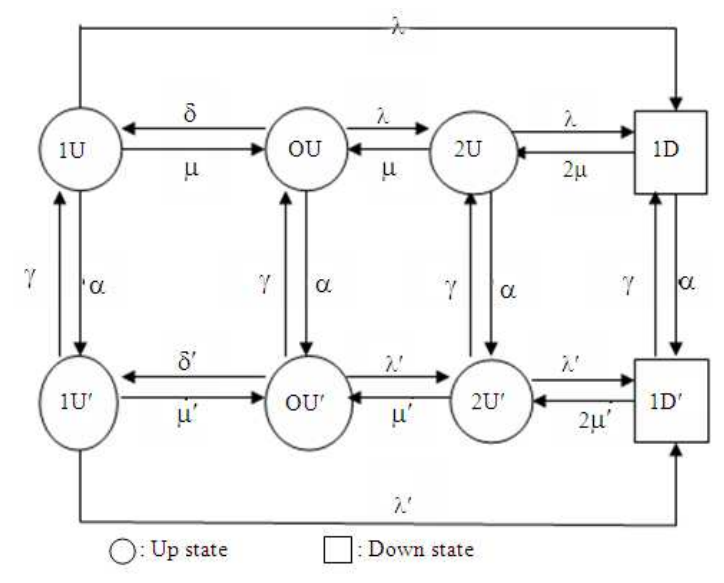

Fig. 2: State transition diagram

- Units are similar and statistically independent

- At time $\mathrm{t}=0$, one unit is operation and the other one is in cold standby for model I and warm standby for model II

- The system operates in changing weather (i.e., normal and stormy weather)

- As soon as the operating unit fails, it is replaced at a certain constant rate by the standby

- The system failure occurs when both the units are non-operative

- A repaired unit is as good as 'new'(this assumption is applicable to Model I and Model II)

Analysis for the two models:

Model I: Deals the reliability analysis of a single-server two-unit cold standby.

System reliability for Model I: The system reliability $\mathrm{R}(\mathrm{t})$ is the probability of failure-free operation of the system in $(0, \mathrm{t})$. To derive an expression for the reliability of the system, we restrict the transitions of the Markov process to the up states, viz.iU[iU'], $\mathrm{i}=0,1$. Using the infinitesimal generator given in the Fig. 1; pertaining to these states and standard probabilistic arguments, we derive the following differential equations:

$$
\begin{aligned}
\frac{\mathrm{dp}_{\mathrm{ou}}}{\mathrm{dt}} & =-(\lambda+\alpha) \mathrm{p}_{\mathrm{ou}}(\mathrm{t})+\mu \mathrm{p}_{1 \mathrm{u}}(\mathrm{t}) \gamma \mathrm{p}_{\mathrm{ou}}(\mathrm{t}) \\
\frac{\mathrm{dp}_{1 \mathrm{U}}}{\mathrm{dt}} & =-(\lambda+\mu+\alpha) \mathrm{p}_{1 \mathrm{U}}(\mathrm{t})+\lambda \mathrm{p}_{\mathrm{oU}}(\mathrm{t})+\gamma \mathrm{p}_{1 \mathrm{U}^{\prime}}(\mathrm{t}) \\
\frac{\mathrm{dP}_{0 \mathrm{U}^{\prime}}}{\mathrm{dt}} & =-\left(\lambda^{\prime}+\gamma\right) \mathrm{P}_{0 \mathrm{U}^{\prime}}(\mathrm{t})+\mu^{\prime} \mathrm{P}_{1 \mathrm{U}^{\prime}}(\mathrm{t})+\alpha \mathrm{p}_{0 \mathrm{U}}(\mathrm{t}) \\
\frac{\mathrm{dp}_{1 \mathrm{U}^{\prime}}}{\mathrm{dt}} & =-\left(\lambda^{\prime}+\mu^{\prime}+\gamma\right) \mathrm{p}_{1 \mathrm{U}^{\prime}}(\mathrm{t})+\lambda^{\prime} \mathrm{p}_{0 \mathrm{U}^{\prime}}(\mathrm{t})+\alpha \mathrm{p}_{1 \mathrm{U}}(\mathrm{t})
\end{aligned}
$$

Taking Laplace transform of $\mathrm{P}_{\mathrm{iu}\left[\mathrm{iu}^{\prime}\right]}^{\prime}(\mathrm{t}), \mathrm{i}=0,1$, on both the sides of the differential Eq. 1 and using the initial conditions at time $\mathrm{t}=0, \mathrm{P}_{\mathrm{ou}}(0)=1$ and all other initial condition probabilities are equal to zero, solving for $\mathrm{P}_{\mathrm{iu}\left[i u^{\prime}\right]}(\mathrm{s})$; we get:

$$
\begin{aligned}
& \mathrm{P}_{\mathrm{OU}}(\mathrm{s})=\frac{\left(\mathrm{s}+\gamma+\lambda^{\prime}\right)\left(\begin{array}{l}
\left.(\mathrm{s}+\alpha+\lambda+\mu)\left(\mathrm{s}+\gamma+\lambda^{\prime}+\mu^{\prime}\right)-\alpha \gamma\right) \\
-\lambda^{\prime}(\mathrm{s}+\alpha+\lambda+\mu)
\end{array}\right)}{\prod_{\mathrm{r}=1}^{4}\left(\mathrm{~s}-\mathrm{s}_{\mathrm{r}}\right)} \\
& \mathrm{P}_{\mathrm{IU}}(\mathrm{s})=\frac{\lambda\left(\mathrm{s}+\gamma+\lambda^{\prime}\right)\left(\mathrm{s}+\gamma+\lambda^{\prime}+\mu^{\prime}\right)-\lambda^{\prime}\left(\lambda \mu^{\prime}-\alpha \gamma\right)}{\prod_{\mathrm{r}=1}^{4}\left(\mathrm{~s}-\mathrm{s}_{\mathrm{r}}\right)} \\
& \mathrm{P}_{\mathrm{OU}}(\mathrm{s})=\frac{\alpha(\mathrm{s}+\alpha+\lambda+\mu)\left(\mathrm{s}+\gamma+\lambda^{\prime}+\mu^{\prime}\right)+\alpha\left(\lambda \mu^{\prime}-\alpha \gamma\right)}{\prod_{\mathrm{r}=1}^{4}\left(\mathrm{~s}-\mathrm{s}_{\mathrm{r}}\right)} \\
& \mathrm{P}_{\mathrm{IU}}(\mathrm{s})=\frac{\alpha\left(\lambda\left(\mathrm{s}+\gamma+\lambda^{\prime}\right)+\lambda^{\prime}(\mathrm{s}+\alpha+\lambda+\mu)\right)}{\prod_{\mathrm{r}=1}^{4}\left(\mathrm{~s}-\mathrm{s}_{\mathrm{r}}\right)}
\end{aligned}
$$

Now taking inverse LT of Eq. 2 we get:

$$
\begin{aligned}
& \mathrm{P}_{\mathrm{OU}}(\mathrm{t})=\sum_{\mathrm{i}=1}^{4}\left[\left(\mathrm{~s}_{\mathrm{i}}+\gamma+\lambda^{\prime}\right)\left(\left(\mathrm{s}_{\mathrm{i}}+\alpha+\lambda+\mu\right)\left(\mathrm{s}_{\mathrm{i}}+\gamma+\lambda^{\prime}+\mu^{\prime}\right)-\alpha \gamma\right)\right. \\
& \left.-\lambda^{\prime}\left(\mathrm{s}_{\mathrm{i}}+\alpha+\lambda+\mu\right) / \prod_{\mathrm{r}=1, \mathrm{r} \neq \mathrm{i}}^{4}\left(\mathrm{~s}_{\mathrm{i}}-\mathrm{s}_{\mathrm{r}}\right)\right] \mathrm{e}^{\mathrm{s}_{\mathrm{i}} \mathrm{t}} \\
& \mathrm{P}_{1 \mathrm{U}}(\mathrm{t})=\sum_{\mathrm{i}=1}^{4} \frac{\lambda\left(\mathrm{s}_{\mathrm{i}}+\gamma+\lambda^{\prime}\right)\left(\mathrm{s}_{\mathrm{i}}+\gamma+\lambda^{\prime}+\mu^{\prime}\right)-\lambda^{\prime}\left(\lambda \mu^{\prime}-\alpha \gamma\right) \mathrm{e}^{\mathrm{s}_{\mathrm{i}} \mathrm{t}}}{\prod_{\mathrm{r}=1, \mathrm{r} \neq \mathrm{i}}^{4}\left(\mathrm{~s}_{\mathrm{i}}-\mathrm{s}_{\mathrm{r}}\right)} \\
& \mathrm{P}_{\mathrm{OU}^{\prime}}(\mathrm{t})= \\
& \sum_{\mathrm{i}=1}^{4} \frac{\alpha\left(\mathrm{s}_{\mathrm{i}}+\alpha+\lambda+\mu\right)\left(\mathrm{s}_{\mathrm{i}}+\gamma+\lambda^{\prime}+\mu^{\prime}\right)+\alpha\left(\lambda \mu^{\prime}-\alpha \gamma\right) \mathrm{e}^{\mathrm{s}_{\mathrm{i}} \mathrm{t}}}{\prod_{\mathrm{r}=1, \mathrm{r} \neq \mathrm{i}}^{4}\left(\mathrm{~s}_{\mathrm{i}}-\mathrm{s}_{\mathrm{r}}\right)} \\
& \mathrm{P}_{1 \mathrm{U}^{\prime}}(\mathrm{t})=\sum_{\mathrm{i}=1}^{4} \frac{\alpha\left(\lambda\left(\mathrm{s}_{\mathrm{i}}+\gamma+\lambda^{\prime}\right)+\lambda^{\prime}\left(\mathrm{s}_{\mathrm{i}}+\alpha+\lambda+\mu\right)\right)}{\prod_{\mathrm{r}=1, \mathrm{r} \neq \mathrm{i}}^{4}\left(\mathrm{~s}_{\mathrm{i}}-\mathrm{s}_{\mathrm{r}}\right)} \mathrm{e}^{\mathrm{s}_{\mathrm{i}} \mathrm{t}}
\end{aligned}
$$

Then the system reliability for Model I is given by:

$$
\begin{aligned}
\mathrm{R}(\mathrm{t})= & \mathrm{P}_{\mathrm{OU}}(\mathrm{t})+\mathrm{P}_{\mathrm{IU}}(\mathrm{t})+\mathrm{P}_{\mathrm{OU}^{\prime}}(\mathrm{t})+\mathrm{P}_{1 \mathrm{U}^{\prime}}(\mathrm{t}) \\
& =\sum_{\mathrm{i}=1}^{4}\left[( \mathrm { s } _ { \mathrm { i } } + \gamma + \lambda ^ { \prime } ) \left(\lambda\left(\mathrm{s}_{\mathrm{i}}+\alpha+\gamma+\lambda^{\prime}+\mu^{\prime}\right)\right.\right. \\
& \left.+\left(-\alpha \gamma+\left(\mathrm{s}_{\mathrm{i}}+\alpha+\lambda+\mu\right)\left(\mathrm{s}_{\mathrm{i}}+\gamma+\lambda^{\prime}+\mu^{\prime}\right)\right)\right) \\
& +\left(\mathrm{s}_{\mathrm{i}}+\alpha+\lambda+\mu\right)\left(-\lambda^{\prime} \mu^{\prime}+\alpha\left(\mathrm{s}_{\mathrm{i}}+\gamma+2 \lambda^{\prime}+\mu^{\prime}\right)\right) \\
& \left.+\left(\lambda \mu^{\prime}-\alpha \gamma\right)\left(\alpha-\lambda^{\prime}\right) \prod_{\mathrm{j}=1, j \neq i}^{4}\left(\mathrm{~s}_{\mathrm{i}}-\mathrm{s}_{\mathrm{j}}\right)\right] \mathrm{e}^{\mathrm{s}_{\mathrm{i}} \mathrm{t}}
\end{aligned}
$$

where, $s_{1}, s_{2}, s_{3}, s_{4}$, the roots of the polynomial for the following equation: 


$$
\begin{aligned}
& \left(-\alpha \gamma(s+\alpha+\lambda+\mu)+\left(s+\gamma+\lambda^{\prime}\right)\right. \\
& (-\lambda \mu+(s+\alpha+\lambda)(s+\alpha+\lambda+\mu))) \\
& \left(s+\gamma+\lambda^{\prime}+\mu^{\prime}\right)-\alpha \gamma\left(-\alpha \gamma+\left(s+\gamma+\lambda^{\prime}\right)\right. \\
& \left.(s+\alpha+\lambda)+\lambda \mu^{\prime}\right)+\lambda^{\prime}(-\alpha \gamma \mu-(-\lambda \mu+(s+\alpha+\lambda) \\
& \left.(s+\alpha+\lambda+\mu)) \mu^{\prime}\right)=0
\end{aligned}
$$

Mean time to system for Model I: The Laplace transform of the reliability of the system for Model I is given by:

$$
\begin{aligned}
\mathrm{R}(\mathrm{s})= & \sum_{\mathrm{i}=0}^{1} \mathrm{P}_{\mathrm{iU}}(\mathrm{s})+\sum_{\mathrm{i}=0}^{1} \mathrm{P}_{\mathrm{i} \mathrm{U}^{\prime}}(\mathrm{s}) \\
& =\left[( \mathrm { s } + \gamma + \lambda ^ { \prime } ) \left(\lambda\left(\mathrm{s}+\alpha+\gamma+\lambda^{\prime}+\mu^{\prime}\right)\right.\right. \\
& \left.+\left(-\alpha \gamma+(\mathrm{s}+\alpha+\lambda+\mu)\left(\mathrm{s}+\gamma+\lambda^{\prime}+\mu^{\prime}\right)\right)\right) \\
& +(\mathrm{s}+\alpha+\lambda+\mu)\left(-\lambda^{\prime} \mu^{\prime}+\alpha\left(\mathrm{s}+\gamma+2 \lambda^{\prime}+\mu^{\prime}\right)\right) \\
& \left.+\left(\lambda \mu^{\prime}-\alpha \gamma\right)\left(\alpha-\lambda^{\prime}\right)\right] / \prod_{\mathrm{r}=1}^{4}\left(\mathrm{~s}-\mathrm{s}_{\mathrm{r}}\right)
\end{aligned}
$$

The mean time to failure of the system for Model I is given by:

$$
\begin{aligned}
\operatorname{MTTF}=\lim _{s \rightarrow 0} R(s)= & {\left[( \gamma + \lambda ^ { \prime } ) \left(\lambda\left(\alpha+\gamma+\lambda^{\prime}+\mu^{\prime}\right)\right.\right.} \\
& \left.+\left(-\alpha \gamma+(\alpha+\lambda+\mu)\left(\gamma+\lambda^{\prime}+\mu^{\prime}\right)\right)\right) \\
& +(\alpha+\lambda+\mu)\left(-\lambda^{\prime} \mu^{\prime}+\alpha\left(\gamma+2 \lambda^{\prime}+\mu^{\prime}\right)\right) \\
& \left.+\left(\lambda \mu^{\prime}-\alpha \gamma\right)\left(\alpha-\lambda^{\prime}\right)\right] / \prod_{\mathrm{r}=1}^{4}\left(-\mathrm{s}_{\mathrm{r}}\right)
\end{aligned}
$$

Where:

$$
\begin{aligned}
\prod_{\mathrm{r}=1}^{4}\left(-\mathrm{s}_{\mathrm{r}}\right)= & {\left[\alpha^{2} \lambda^{\prime 2}+\alpha \lambda^{\prime}\left(2 \gamma \lambda+\lambda^{\prime}(2 \lambda+\mu)\right)\right.} \\
& \left.+\lambda^{2}\left(\gamma^{2}+\lambda^{\prime 2}+\gamma\left(2 \lambda^{\prime}+\mu^{\prime}\right)\right)\right]
\end{aligned}
$$

Model II: Deals the reliability analysis of a singleserver two-unit warm standby Fig. 2 shows the states of the system for Model II.

System reliability for Model II: The system reliability $R(t)$ is the probability of failure-free operation of the system in $(0, \mathrm{t})$. To derive an expression for the reliability of the system, we restrict the transitions of the Markov process to the up states, viz.iU[iU'], $\mathrm{i}=0,1,2$. Using the infinitesimal generator given in the Fig. 2 pertaining to these states and standard probabilistic arguments, we derive the following differential equations:

$$
\begin{aligned}
\frac{d p_{\mathrm{OU}}}{\mathrm{dt}}= & -(\delta+\lambda+\alpha) \mathrm{p}_{\mathrm{OU}}(\mathrm{t})+\mu \mathrm{p}_{1 \mathrm{U}}(\mathrm{t})+\mu \mathrm{p}_{2 \mathrm{U}}(\mathrm{t})+\gamma \mathrm{p}_{\mathrm{OU}}(\mathrm{t}) \\
\frac{\mathrm{d} \mathrm{p}_{1 \mathrm{U}}}{\mathrm{dt}}= & -(\mu+\lambda+\alpha) \mathrm{p}_{1 \mathrm{U}}(\mathrm{t})+\delta \mathrm{p}_{0 \mathrm{U}}(\mathrm{t})+\gamma \mathrm{p}_{1 \mathrm{U}^{\prime}}(\mathrm{t}) \\
\frac{\mathrm{dp} \mathrm{p}_{2 \mathrm{U}}}{\mathrm{dt}}= & -(\lambda+\mu+\alpha) \mathrm{p}_{2 \mathrm{U}}(\mathrm{t})+\lambda \mathrm{p}_{0 \mathrm{U}}(\mathrm{t})+\gamma \mathrm{p}_{2 \mathrm{U}}{ }^{\prime}(\mathrm{t}) \\
\frac{\mathrm{dp} \mathrm{oU}^{\prime}}{\mathrm{dt}}= & -\left(\delta^{\prime}+\lambda^{\prime}+\gamma\right) \mathrm{p}_{\mathrm{OU}}(\mathrm{t})+\mu^{\prime} \mathrm{p}_{1 \mathrm{U}^{\prime}}(\mathrm{t}) \\
& +\mu^{\prime} \mathrm{p}_{2 \mathrm{U}^{\prime}}(\mathrm{t})+\alpha \mathrm{p}_{\mathrm{OU}}(\mathrm{t}) \\
\frac{\mathrm{dp} \mathrm{p}_{1 \mathrm{U}^{\prime}}}{\mathrm{dt}}= & -\left(\mu^{\prime}+\lambda^{\prime}+\gamma\right) \mathrm{p}_{1 \mathrm{U}^{\prime}}(\mathrm{t})+\delta^{\prime} \mathrm{p}_{\mathrm{OU}}(\mathrm{t})+\alpha \mathrm{p}_{1 \mathrm{U}}(\mathrm{t}) \\
\frac{\mathrm{d} \mathrm{p}_{2 \mathrm{U}^{\prime}}}{\mathrm{dt}}= & -\left(\lambda^{\prime}+\mu^{\prime}+\gamma\right) \mathrm{p}_{2 \mathrm{U}^{\prime}}(\mathrm{t})+\lambda^{\prime} \mathrm{p}_{0 \mathrm{U}^{\prime}}(\mathrm{t})+\alpha \mathrm{p}_{2 \mathrm{U}}(\mathrm{t})
\end{aligned}
$$

Taking Laplace transform of $\mathrm{P}_{\text {iu }\left[\mathrm{iu}^{\prime}\right]}^{\prime}(\mathrm{t}), \mathrm{i}=0,1,2$, on both the sides of the differential Eq. 7 and using the initial conditions at time $\mathrm{t}=0 \mathrm{P}_{\mathrm{ou}}(0)=1$ and all other initial condition probabilities are equal to zero, solving for $\mathrm{P}_{\text {iu }[\text { iu'] }}(\mathrm{s})$; we get:

$$
\begin{aligned}
& \mathrm{P}_{\mathrm{OU}}(\mathrm{s})=\frac{\mathrm{J}_{3}(\mathrm{~s})-\alpha \gamma \mathrm{J}_{2}(\mathrm{~s})}{\prod_{\mathrm{r}=1}^{6}\left(\mathrm{~s}-\mathrm{s}_{\mathrm{r}}\right)} \\
& \mathrm{P}_{\mathrm{OU}}(\mathrm{s})=\frac{\left(\mathrm{s}+\gamma+\lambda^{\prime}+\mu^{\prime}\right) \mathrm{J}_{6}(\mathrm{~s})-\delta^{\prime} \mathrm{J}_{5}(\mathrm{~s})}{\prod_{\mathrm{r}=1}^{6}\left(\mathrm{~s}-\mathrm{s}_{\mathrm{r}}\right)}
\end{aligned}
$$$$
\mathrm{P}_{\mathrm{IU}}(\mathrm{s})=\frac{\delta\left(\mathrm{s}+\gamma+\lambda^{\prime}+\mu^{\prime}\right) \mathrm{J}_{4}(\mathrm{~s})-\delta^{\prime} \mathrm{J}_{5}(\mathrm{~s})}{\prod_{\mathrm{r}=1}^{6}\left(\mathrm{~s}-\mathrm{s}_{\mathrm{r}}\right)}
$$

$$
\mathrm{P}_{1 \mathrm{U}^{\prime}}(\mathrm{s})=\frac{\delta^{\prime} \mathrm{J}_{7}(\mathrm{~s})+\alpha \delta \mathrm{J}_{4}(\mathrm{~s})}{\prod_{\mathrm{r}=1}^{6}\left(\mathrm{~s}-\mathrm{s}_{\mathrm{r}}\right)}
$$

$$
\mathrm{P}_{2 \mathrm{U}}(\mathrm{s})=\frac{\lambda\left(\mathrm{s}+\gamma+\lambda^{\prime}+\mu^{\prime}\right) \mathrm{J}_{2}(\mathrm{~s})-\lambda^{\prime} \mathrm{J}_{5}(\mathrm{~s})}{\prod_{\mathrm{r}=1}^{6}\left(\mathrm{~s}-\mathrm{s}_{\mathrm{r}}\right)}
$$$$
\mathrm{P}_{2 \mathrm{U}^{\prime}}(\mathrm{s})=\frac{\lambda^{\prime} \mathrm{J}_{6}(\mathrm{~s})+\alpha \lambda \mathrm{J}_{2}(\mathrm{~s})}{\prod_{\mathrm{r}=1}^{6}\left(\mathrm{~s}-\mathrm{s}_{\mathrm{r}}\right)}
$$

Now taking inverse LT of Eq. 8 we get: 


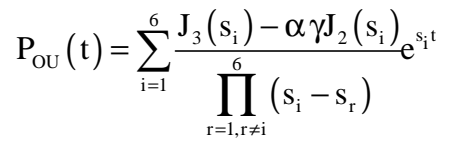

$$
\begin{aligned}
& \mathrm{P}_{1 \mathrm{U}}(\mathrm{t})=\sum_{\mathrm{i}=1}^{6} \frac{\delta\left(\mathrm{s}_{\mathrm{i}}+\gamma+\lambda^{\prime}+\mu^{\prime}\right) \mathrm{J}_{4}\left(\mathrm{~s}_{\mathrm{i}}\right)-\delta^{\prime} \mathrm{J}_{5}\left(\mathrm{~s}_{\mathrm{i}}\right) \mathrm{e}^{\mathrm{s}_{\mathrm{i}} \mathrm{t}}}{\prod_{\mathrm{r}=1, \mathrm{r} \neq \mathrm{i}}^{6}\left(\mathrm{~s}_{\mathrm{i}}-\mathrm{s}_{\mathrm{r}}\right)} \\
& \mathrm{P}_{2 \mathrm{U}}(\mathrm{t})=\sum_{\mathrm{i}=1}^{6} \frac{\lambda\left(\mathrm{s}_{\mathrm{i}}+\gamma+\lambda^{\prime}+\mu^{\prime}\right) \mathrm{J}_{2}\left(\mathrm{~s}_{\mathrm{i}}\right)-\lambda^{\prime} \mathrm{J}_{5}\left(\mathrm{~s}_{\mathrm{i}}\right) \mathrm{e}^{\mathrm{s}_{\mathrm{i}} \mathrm{t}}}{\prod_{\mathrm{r}=1, \mathrm{r} \neq \mathrm{i}}^{6}\left(\mathrm{~s}_{\mathrm{i}}-\mathrm{s}_{\mathrm{r}}\right)} \\
& \mathrm{P}_{\mathrm{OU}}(\mathrm{t})=\sum_{\mathrm{i}=1}^{6} \frac{\left(\mathrm{s}_{\mathrm{i}}+\gamma+\lambda^{\prime}+\mu^{\prime}\right) \mathrm{J}_{6}\left(\mathrm{~s}_{\mathrm{i}}\right)-\alpha \mathrm{J}_{5}\left(\mathrm{~s}_{\mathrm{i}}\right)}{\prod_{\mathrm{r}=1, \mathrm{r} \neq \mathrm{i}}^{6}\left(\mathrm{~s}_{\mathrm{i}}-\mathrm{s}_{\mathrm{r}}\right)} \mathrm{e}^{\mathrm{s}_{\mathrm{i}} \mathrm{t}} \\
& \mathrm{P}_{1 \mathrm{U}^{\prime}}(\mathrm{t}) \sum_{\mathrm{i}=1}^{6} \frac{\delta^{\prime} \mathrm{J}_{7}\left(\mathrm{~s}_{\mathrm{i}}\right)+\alpha \delta \mathrm{J}_{4}\left(\mathrm{~s}_{\mathrm{i}}\right)^{\mathrm{s}_{\mathrm{i}} \mathrm{t}}}{\prod_{\mathrm{r}=1, \mathrm{r} \neq \mathrm{i}}^{6}\left(\mathrm{~s}_{\mathrm{i}}-\mathrm{s}_{\mathrm{r}}\right)} \\
& \mathrm{P}_{2 \mathrm{U}^{\prime}}(\mathrm{t})=\sum_{\mathrm{i}=1}^{6} \frac{\lambda^{\prime} \mathrm{J}_{6}\left(\mathrm{~s}_{\mathrm{i}}\right)+\alpha \lambda \mathrm{J}_{2}\left(\mathrm{~s}_{\mathrm{i}}\right)^{\mathrm{s}_{\mathrm{i}} \mathrm{t}}}{\prod_{\mathrm{r}=1, \mathrm{r} \neq \mathrm{i}}^{6}\left(\mathrm{~s}_{\mathrm{i}}-\mathrm{s}_{\mathrm{r}}\right)}
\end{aligned}
$$

Then the system reliability is given by:

$$
\begin{aligned}
\mathrm{R}(\mathrm{t})= & \sum_{\mathrm{i}=0}^{2} \mathrm{P}_{\mathrm{iU}}(\mathrm{t})+\sum_{\mathrm{i}=0}^{2} \mathrm{P}_{\mathrm{iU}}{ }^{\prime}(\mathrm{t}) \\
= & \sum_{\mathrm{i}=1}^{6}\left[\left(\lambda\left(\mathrm{s}_{\mathrm{i}}+\alpha+\gamma+\lambda^{\prime}+\mu^{\prime}\right)-\alpha \lambda\right)\right. \\
& \mathrm{J}_{2}\left(\mathrm{~s}_{\mathrm{i}}\right)+\mathrm{J}_{3}\left(\mathrm{~s}_{\mathrm{i}}\right)+\delta\left(\mathrm{s}_{\mathrm{i}}+\alpha+\gamma+\lambda^{\prime}+\mu^{\prime}\right) \mathrm{J}_{4}\left(\mathrm{~s}_{\mathrm{i}}\right) \\
& -\left(\delta^{\prime}+\lambda^{\prime}-\alpha\right) \mathrm{J}_{5}\left(\mathrm{~s}_{\mathrm{i}}\right)+\left(\mathrm{s}_{\mathrm{i}}+\gamma+2 \lambda^{\prime}+\mu^{\prime}\right) \mathrm{J}_{6}\left(\mathrm{~s}_{\mathrm{i}}\right) \\
& \left.+\delta^{\prime} \mathrm{J}_{7}\left(\mathrm{~s}_{\mathrm{i}}\right) / \prod_{\mathrm{r}=1, \mathrm{r} \neq \mathrm{i}}^{6}\left(\mathrm{~s}_{\mathrm{i}}-\mathrm{s}_{\mathrm{r}}\right)\right] \mathrm{e}^{\mathrm{s}_{\mathrm{i}} \mathrm{t}}
\end{aligned}
$$

Mean time to system for Model II: The Laplace transform of the reliability of the system is given by:

$$
\begin{aligned}
\mathrm{R}(\mathrm{s})= & \sum_{\mathrm{i}=0}^{2} \mathrm{P}_{\mathrm{iU}}(\mathrm{s})+\sum_{\mathrm{i}=0}^{2} \mathrm{P}_{\mathrm{iU}}{ }^{\prime}(\mathrm{s}) \\
& =\left[\left(\lambda\left(\mathrm{s}+\alpha+\gamma+\lambda^{\prime}+\mu^{\prime}\right)-\alpha \lambda\right) \mathrm{J}_{2}(\mathrm{~s})+\mathrm{J}_{3}(\mathrm{~s})+\right. \\
& \delta\left(\mathrm{s}+\alpha+\gamma+\lambda^{\prime}+\mu^{\prime}\right) \mathrm{J}_{4}(\mathrm{~s})-\left(\delta^{\prime}+\lambda^{\prime}-\alpha\right) \mathrm{J}_{5}(\mathrm{~s}) \\
& \left.+\left(\mathrm{s}+\gamma+2 \lambda^{\prime}+\mu^{\prime}\right) \mathrm{J}_{6}(\mathrm{~s})+\delta^{\prime} \mathrm{J}_{7}(\mathrm{~s})\right] \prod_{\mathrm{r}=1, \mathrm{r} \neq \mathrm{i}}^{6}\left(\mathrm{~s}_{\mathrm{i}}-\mathrm{s}_{\mathrm{r}}\right)
\end{aligned}
$$
by:

The mean time to failure of the system is given

$$
\begin{aligned}
& \mathrm{MTTF}=\lim _{s \rightarrow 0} \mathrm{R}(\mathrm{s})= \\
& {\left[\alpha\left(\lambda^{\prime}+\mu^{\prime}\right)+(\lambda+\mu)\left(\gamma+\lambda^{\prime}+\mu^{\prime}\right)\right)\left(\alpha^{2}\left(\delta^{\prime}+2 \lambda^{\prime}+\mu^{\prime}\right)\right.} \\
& +(\delta+2 \lambda+\mu)\left(\gamma^{2}+\lambda^{\prime}\left(\delta^{\prime}+\lambda^{\prime}\right)+\gamma\left(\delta^{\prime}+2 \lambda^{\prime}+\mu^{\prime}\right)\right) \\
& +\alpha\left(\lambda^{\prime}\left(\delta^{\prime}+\lambda^{\prime}+3 \lambda+2 \mu\right)+(2 \lambda+\mu)\left(\delta^{\prime}+\mu^{\prime}\right)\right. \\
& \left.\left.+\delta\left(\gamma^{\prime}+\lambda^{\prime}+\mu^{\prime}\right)+\gamma\left(\delta+\delta^{\prime}+2 \lambda^{\prime}+2 \lambda+\mu+\mu^{\prime}\right)\right)\right] \\
& /\left[\left(\alpha^{2} \lambda^{\prime}\left(\delta^{\prime}+\lambda^{\prime}\right)+\left(\alpha\left(\lambda^{\prime}+\mu^{\prime}\right)+(\lambda+\mu)\left(\gamma+\lambda^{\prime}+\mu^{\prime}\right)\right)\right.\right. \\
& \alpha\left(\gamma\left(\delta \lambda^{\prime}+\lambda\left(\delta^{\prime}+2 \lambda^{\prime}\right)\right)+\lambda^{\prime}\left(\delta^{\prime}+\lambda^{\prime}\right)\right. \\
& \left.\lambda(\delta+\lambda)\left(\gamma^{2}+\lambda^{\prime}\left(\delta^{\prime}+\lambda^{\prime}\right)+\gamma\left(\delta^{\prime}+2 \lambda^{\prime}+\mu^{\prime}\right)\right)\right]
\end{aligned}
$$

Where:

$$
\begin{aligned}
\mathrm{J}_{2}(\mathrm{~s})= & \left(-\delta^{\prime} \mu^{\prime}(\mathrm{s}+\alpha+\lambda+\mu)+\left(\mathrm{s}+\gamma+\delta^{\prime}+\lambda^{\prime}\right)\right. \\
& (-\alpha \gamma(\mathrm{s}+\alpha+\lambda+\mu) \\
\mathrm{J}_{3}(\mathrm{~s})= & (\mathrm{s}+\alpha+\lambda+\mu)\left(-\lambda^{\prime} \mu^{\prime}(-\alpha \gamma+(\mathrm{s}+\alpha+\lambda+\mu)\right. \\
& \left.\left.\left(\mathrm{s}+\gamma+\lambda^{\prime}+\mu^{\prime}\right)\right)+\left(\mathrm{s}+\gamma+\lambda^{\prime}+\mu^{\prime}\right) \mathrm{J}_{2}(\mathrm{~s})\right) \\
\mathrm{J}_{4}(\mathrm{~s})= & \left(-\lambda^{\prime} \mu^{\prime}(\mathrm{s}+\alpha+\lambda+\mu)+\left(\mathrm{s}+\gamma+\delta^{\prime}+\lambda^{\prime}\right)\right. \\
& \left(-\alpha \gamma+(\mathrm{s}+\alpha+\lambda+\mu) \mathrm{J}_{5}(\mathrm{~s})=((\mathrm{s}+\alpha+\lambda+\mu)\right. \\
& \left.\left(\mathrm{s}+\gamma+\lambda^{\prime}+\mu^{\prime}\right)\left(\delta \mu^{\prime}-\alpha \gamma\right)-\alpha \gamma\left(-\alpha \gamma+\delta \mu^{\prime}+\lambda \mu^{\prime}\right)\right) \\
\mathrm{J}_{6}(\mathrm{~s})= & (\mathrm{s}+\alpha+\lambda+\mu)\left(\alpha(\mathrm{s}+\alpha+\lambda+\mu)\left(\mathrm{s}+\gamma+\lambda^{\prime}+\mu^{\prime}\right)\right. \\
& \left.+\alpha\left(\delta \mu^{\prime}-\alpha \gamma\right)\right), \mathrm{J}_{7}(\mathrm{~s})=(\mathrm{s}+\alpha+\lambda+\mu) \\
& \left(\alpha(\mathrm{s}+\alpha+\lambda+\mu)\left(\mathrm{s}+\gamma+\lambda^{\prime}+\mu^{\prime}\right)+\alpha\left(\lambda \mu^{\prime}-\alpha \gamma\right)\right) \\
\mathrm{J}_{\mathrm{r}}(\mathrm{s})= & \mathrm{J}_{\mathrm{r}}\left(\mathrm{s}_{\mathrm{i}}\right) \Leftrightarrow \mathrm{s}=\mathrm{s}_{\mathrm{i}} \forall \mathrm{J}_{\mathrm{r}}(\mathrm{s}), \mathrm{r}=1,2, \ldots, 7
\end{aligned}
$$

and $\mathrm{s}_{1}, \mathrm{~s}_{2}, \ldots, \mathrm{s}_{6}$ the roots of the polynomial for expand the determinant of the following matrix:

$\left[\begin{array}{cccccc}\left(\mathrm{s}+\mathrm{w}_{1}\right) & -\mu & -\mu & -\gamma & 0 & 0 \\ -\delta & \left(\mathrm{s}+\mathrm{w}_{2}\right) & 0 & 0 & -\gamma & 0 \\ -\lambda & 0 & \left(\mathrm{~s}+\mathrm{w}_{2}\right) & 0 & 0 & -\gamma \\ -\alpha & 0 & 0 & \left(\mathrm{~s}+\mathrm{w}_{3}\right) & -\mu^{\prime} & -\mu^{\prime} \\ 0 & -\alpha & 0 & -\delta^{\prime} & \left(\mathrm{s}+\mathrm{w}_{4}\right) & 0 \\ 0 & 0 & -\alpha & -\lambda^{\prime} & 0 & \left(\mathrm{~s}+\mathrm{w}_{4}\right)\end{array}\right]$

where:

$$
\begin{aligned}
& \mathrm{w}_{1}=\delta+\lambda+\alpha \\
& \mathrm{w}_{2}=\mu+\lambda+\alpha \\
& \mathrm{w}_{3}=\delta^{\prime}+\lambda^{\prime}+\gamma \\
& \mathrm{w}_{4}=\lambda^{\prime}+\mu^{\prime}+\gamma
\end{aligned}
$$

Graphical representation: Setting $\mu^{\prime}=0.02$, $\mu=0.03, \gamma=0.06, \lambda^{\prime}=0.03, \alpha=0.04, \quad \delta=0.03, \delta^{\prime}=0.04 \quad$ in Eq. 6 and 12. We get compare between two models. 


\section{CONCLUSION}

First represent Mean Time To Failure (MTTF) and steady state availability for different values of constant failure rate operative unit in normal $(\lambda)$. Also, second represents Mean Time To Failure (MTTF) and steady state availability for different values of constant failure rate operative unit in stormy $\left(\lambda^{\prime}\right)$ of the two Models.

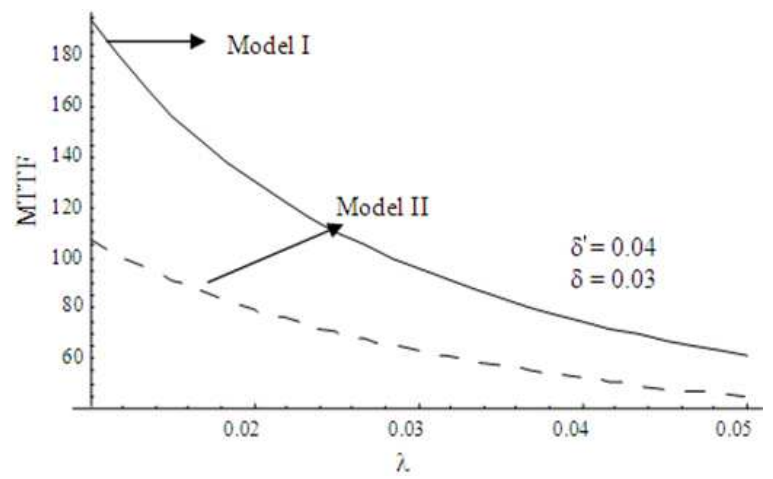

Fig. 3: Represent relation between $\lambda$ and MTTF

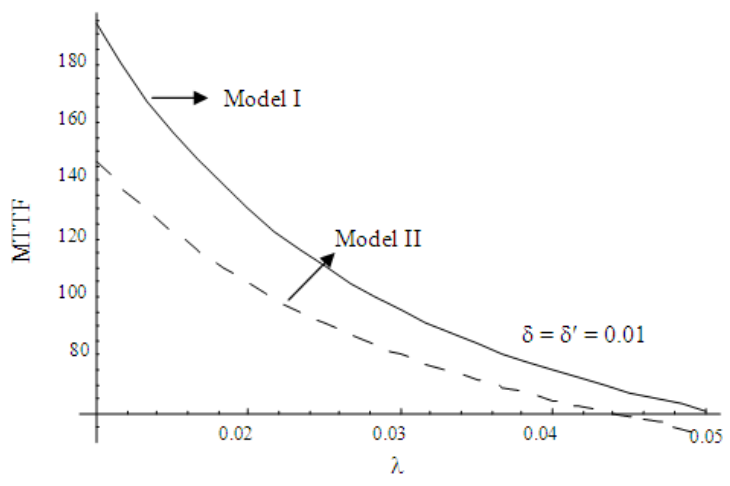

Fig. 4: Represent relation between $\lambda$ and MTTF

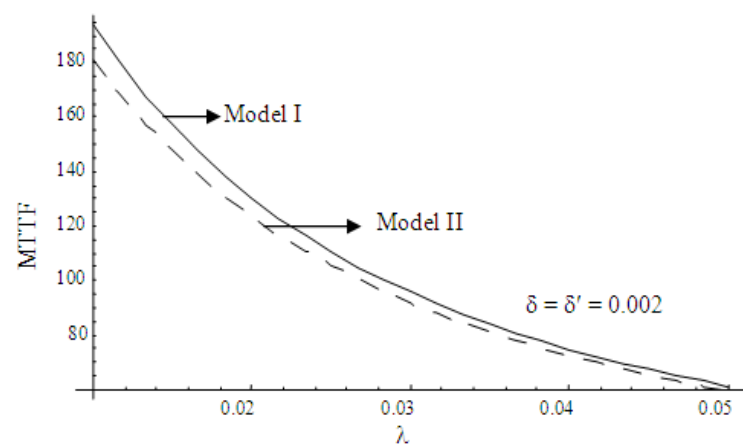

Fig. 5: Represent relation between $\lambda$ and MTT
The Table 1 shows that the present of an using cold standby $\lambda^{\prime}$ leads to improve the values of the mean time to system failure and the steady state availability as shown from their behaviors when plotted against $\lambda$ and $\lambda^{\prime}$.

Figure 3-8 demonstrate the following results which are only to be expected.

Changeover parameter at $\delta\left[\delta^{\prime}\right]$ unit constant failure rate worm standby unit in normal (stormy) weather, are large and the $\lambda\left[\lambda^{\prime}\right]$ unit constant failure rate operative unit in normal (stormy) weather, are small. Then MTTF for Model I is patter than MTTF for Model II.

Changeover parameter at $\delta\left[\delta^{\prime}\right]$ unit constant failure rate warm standby unit in normal (stormy) weather, are equal zero. Then MTTF for Model I is equal to MTTF for Model II.

Table 1: Compare between two models

\begin{tabular}{lll}
\hline & MTTF for Model I & MTTF for Model II \\
\hline $\boldsymbol{\lambda}^{\prime}=\mathbf{0 . 0 3}, \boldsymbol{\lambda}$ & & \\
0.01 & 194.118 & 107.273 \\
0.02 & 130.208 & 79.5066 \\
0.03 & 95.6246 & 63.1030 \\
0.04 & 74.7696 & 52.2857 \\
0.05 & 61.0610 & 44.6221 \\
0.06 & 51.4502 & 38.9112 \\
0.07 & 44.3759 & 34.4924 \\
0.08 & 38.9687 & 30.9724 \\
0.09 & 34.7107 & 28.1029 \\
$\boldsymbol{\lambda}=\mathbf{0 . 0 2}, \boldsymbol{\lambda}^{\prime}$ & & \\
0.01 & 224.740 & 115.686 \\
0.02 & 164.881 & 93.3333 \\
0.03 & 130.208 & 79.5066 \\
0.04 & 108.333 & 70.1149 \\
0.05 & 93.5133 & 63.3221 \\
0.06 & 82.9023 & 58.1818 \\
0.07 & 74.9718 & 54.1573 \\
0.08 & 68.8406 & 50.9213 \\
0.09 & 63.9695 & 48.2630 \\
\hline
\end{tabular}

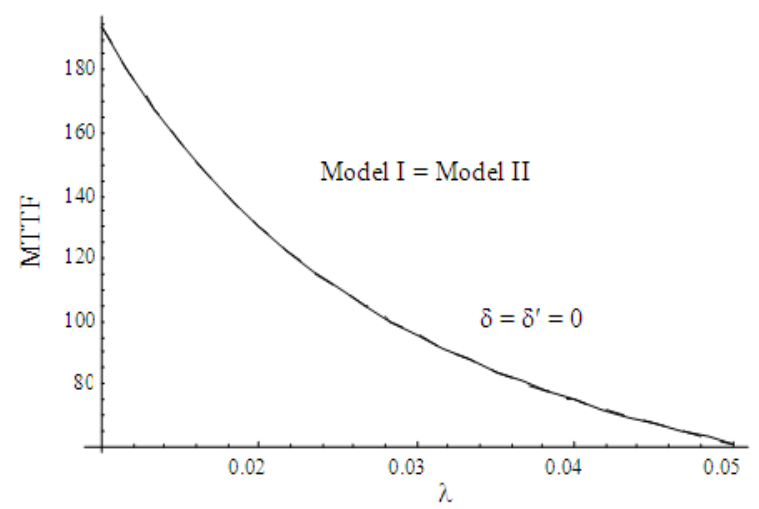

Fig. 6: Represent relation between $\lambda$ and MTTF 


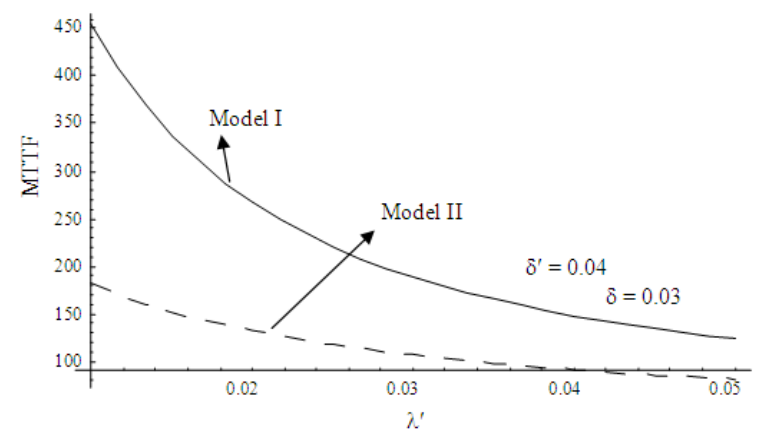

Fig. 7: Represent relation between $\lambda^{\prime}$ and MTTF

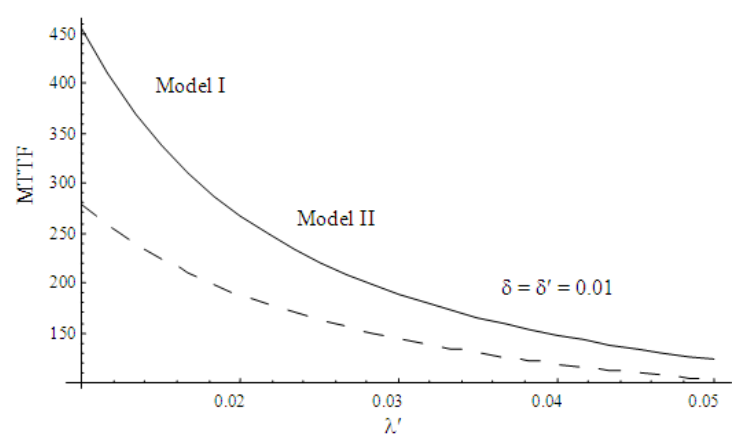

Fig. 8: Represent relation between $\lambda^{\prime}$ and MTTF

\section{REFERENCES}

Balbir, S.D. and N.R. Subramanyam, 1985. Stochastic analysis of two-unit outdoor electric power systems in changing weather. Microelect. Reliab., 25: 357367.

http://cat.inist.fr/?aModele $=$ afficheN\&cpsidt $=8476$ 978

Dhillon, B.S. and Natesan, 1986. Reliability evaluation of electric power systems in alternating environment. Microelectr. Reliab., 26: 679-690.
Kuo-Hsiung Wang, Wen-LI Dong and Jyh-Bin Ke, 2006. Comparison of reliability and the availability between four systems with warm standby components and standby switching failure. Applied Math. $\quad$ Comput., 28: 17-21. http://cat.inist.fr $/$ ?aModele $=$ afficheN\&cpsidt $=1844$ 2281

Mokaddis, G.S., M. Salah EL-Sherbeny and Y. Mahmoud Ayid, 2009. Stochastic behavior of a two-unit warm standby system with two types of repairmen and patience time. J. Math. Stat., 5: 42-46. http://www.scipub.org/fulltext/jms2/jms2514246.pdf 